\title{
Tofacitinib: una revisión de su uso en pacientes con colitis ulcerosa
}

\section{Tofacitinib: a review of its use in patients with ulcerous colitis}

\author{
José A. Romero-Lozanía ${ }^{1 *}$, Korely C. Trujillo-de la Fuente ${ }^{1}$, Rebeca Pérez-Cabeza de Vaca ${ }^{2}$ y \\ Tomás Cortés-Espinosa ${ }^{1}$ \\ ${ }^{1}$ Centro de Enfermedad Inflamatoria Intestinal; ${ }^{2}$ División de Investigación Biomédica. Centro Médico Nacional 20 de Noviembre, Ciudad de México, México
}

\begin{abstract}
Resumen
El tofacitinib es el único inhibidor de JAK autorizado para el tratamiento de la colitis ulcerosa (CU) y aprobado por la Food and Drug Administration y la European Medicines Agency desde 2018. Es un paninhibidor de JAK que inhibe preferentemente la señalización de los receptores de citocinas que se asocian con JAK1 y JAK3, lo que imposibilita la fosforilación de JAK necesaria para el acoplamiento de STAT, impidiendo así su traslocación hacia el núcleo y la transcripción de proteínas inflamatorias. El tofacitinib oral está indicado para el tratamiento de la CU de moderada a grave refractaria o intolerante a los anti-TNF. Demostró su efectividad en los ensayos OCTAVE, pero debido a los amplios efectos inmunosupresores existe preocupación por la presentación de eventos adversos como infecciones graves, infecciones por herpes zóster, neoplasias malignas, cáncer de piel no melanoma, tromboembolias y eventos cardiovasculares mayores. Las revisiones sistemáticas muestran que la frecuencia de eventos adversos es similar con tofacitinib y con placebo. Los inhibidores de JAK son una nueva opción terapéutica eficaz, segura y que además tiene ventajas sobre la terapia biológica, como son unos menores costos de producción, su fácil administración oral y no generar inmunogenicidad.
\end{abstract}

Palabras clave: Vía JAK-STAT. Inhibidor JAK1 / JAK3. Tofacitinib. Colitis ulcerosa. Falla a anti-TNF. Intolerancia a anti-TNF.

\section{Abstract}

Tofacitinib is the only JAK inhibitor licensed for the treatment of ulcerative colitis (UC) and approved by the Food and Drug Administration and the European Medicines Agency since 2018. It is a pan-JAK inhibitor that preferentially inhibits the signaling of cytokine receptors that are associated with JAK1 and JAK3, which makes impossible the phosphorylation of JAK necessary for the coupling of STAT, thus preventing its translocation into the nucleus and the transcription of inflammatory proteins. It demonstrated its effectiveness and good safety profile in the OCTAVE trials, but due to the broad immunosuppressive effects there is concern about the presentation of adverse events such as serious infections, herpes zoster infections, malignant neoplasms, non-melanoma skin cancer, thromboembolism, and major cardiovascular events. Systematic reviews show that the frequency of adverse events is similar with tofacitinib and placebo. JAK inhibitors are a new therapeutic option that is effective, safe, and also has advantages over biological therapy, such as lower production costs, easy oral administration and no immunogenicity.

Keywords: JAK-STAT pathway. JAK1/JAK3 inhibitor. Tofacitinib. Ulcerative colitis. Anti-TNF failure. Anti-TNF intolerance.

Correspondencia:

*Korely C. Trujillo-de la Fuente

E-mail: dra.korely.trujillo@ hotmail.com
Disponible en internet: 22-02-2022

Fecha de aceptación: 03-11-2021

DOI: 10.24875/IMIDS.M21000015
Rev Mex Enferm Inflam Inmunomed.2021;95(4):112-122

www.IMIDsMexico.com 


\section{Introducción}

El arsenal terapéutico para la enfermedad inflamatoria intestinal (EII) ha aumentado considerablemente en las últimas décadas. En la actualidad se encuentran disponibles varias alternativas, entre las que se encuentran esteroides, aminosalicilatos, inmunosupresores y anticuerpos contra el factor de necrosis tumoral alfa (anti-TNF- $\alpha$ ), antiintegrina $\alpha 4 \beta 7 \mathrm{y}$ antiinterleucinas $12 / 23^{1,2}$. A pesar del beneficio de estas estrategias, algunos pacientes seguirán sin responder o perderán la respuesta a las terapias actuales conforme pase el tiempo, por lo que se están considerando nuevos enfoques que interfieren con la vía JAK (Janus cinasa)-STAT (transductor de señal y activador de proteínas de transcripción $)^{3}$.

Esta diversidad de objetivos terapéuticos sugiere que la patogenia subyacente a la Ell es compleja y depende de varias vías moleculares ${ }^{4}$. Las vías JAKSTAT median los efectos biológicos de más de 50 citocinas y factores de crecimiento, y muchos de estos efectos biológicos tienen funciones fundamentales tanto en la homeostasis intestinal como en la Ell ${ }^{3}$.

El objetivo de este artículo es hacer una revisión de la evidencia científica acerca del uso del tofacitinib en la colitis ulcerosa (CU).

\section{La vía JAK-STAT y su inhibición}

Hay tres partes clave de la vía JAK-STAT: los receptores (a los cuales se unen las señales químicas), la enzimas JAK (se encuentran asociadas a la porción intracelular de los receptores) y los STAT (transducen la señal desde los receptores y posteriormente activan la transcripción de proteínas a nivel nuclear $)^{5,6}$ (Fig. 1).

Las enzimas JAK se identificaron entre 1989 y 1993, y fueron llamadas así por el dios romano de dos caras, Janus $^{7-11}$. La familia de las JAK está constituida por cuatro miembros distintos: JAK1, JAK2, JAK3 y tirosina cinasa 2 (TYK2) $)^{3,12}$. La familia STAT está integrada por siete miembros: STAT1, STAT2, STAT3, STAT4, STAT5a, STAT5b y STAT6 ${ }^{13,14}$.

Los inhibidores de la JAK (iJAK) son una nueva clase de fármacos de molécula pequeña (menos de $1 \mathrm{kDa}$ ) que modulan las vías inflamatorias bloqueando uno o más receptores acoplados a JAK y se utilizan cada vez más en el tratamiento de enfermedades inmunomediadas $^{15}$. Algunos de ellos bloquean todas los JAK con potencia similar (paninhibidores de JAK), mientras que otros son más selectivos. Sin embargo, se cree que una mayor selectividad se asocia con menos eventos adversos, pero también con menos eficacia ${ }^{16}$.

\section{Tofacitinib}

Hasta la fecha, el tofacitinib es el único iJAK aprobado desde 2018 por la Food and Drug Administration (FDA) de los Estados Unidos de América y la European Medicines Agency (EMA) para el tratamiento de la CU de moderada a grave que es refractaria o intolerante a los anti-TNF ${ }^{17-20}$. Otras moléculas iJAK, como filgotinib (GLPG0634), upadacitinib (ABT-494), izencitinib (D-1473), ritlecitinib (Pf-06651600), brepocitinib (Pf-0670084) y deucravacitinib (BMS-986165), se encuentran en ensayos clínicos de fase 2-3 para el tratamiento de la $\mathrm{CU}^{21-30}$ (Tabla 2).

La aprobación del tofacitinib se basó en los resultados de los ensayos OCTAVE, que consistieron en dos ensayos de inducción (OCTAVE 1 y 2) y un ensayo de mantenimiento (OCTAVE Sustain) ${ }^{31}$.

En los ensayos de inducción OCTAVE 1 y 2 se incluyeron pacientes con CU activa de moderada a grave a pesar de ser tratados con terapia convencional previa o terapia con anti-TNF, los cuales fueron asignados aleatoriamente para recibir terapia de inducción con tofacitinib (10 $\mathrm{mg}$ dos veces al día) o placebo durante 8 semanas. En el ensayo OCTAVE 1, la remisión a las 8 semanas se produjo en el $18.5 \%$ de los pacientes del grupo con tofacitinib, frente al $8.2 \%$ del grupo con placebo $(p=0.007)$. En el ensayo OCTAVE 2 , la remisión se produjo en el $16.6 \%$ de los pacientes con tofacitinib, frente al $3.6 \%(p<0.001)$ en los pacientes con placebo $^{18}$.

En el ensayo de mantenimiento OCTAVE Sustain se incluyeron pacientes de los ensayos OCTAVE 1 y 2 que lograron respuesta clínica a la terapia de inducción. Fueron asignados al azar para recibir terapia de mantenimiento con tofacitinib $(5 \mathrm{mg} \circ 10 \mathrm{mg}$ dos veces al día) o placebo durante 52 semanas. La remisión a las 52 semanas se produjo en el $34.3 \%$ de los pacientes en el grupo de $5 \mathrm{mg}$ de tofacitinib y en el $40.6 \%$ en el grupo de $10 \mathrm{mg}$ de tofacitinib, frente al $11.1 \%$ en el grupo de placebo $(p<0.001$ para ambas comparaciones con placebo). No se encontró diferencia estadísticamente significativa en la remisión entre los grupos de tofacitinib ${ }^{18}$.

Aunque no existen ensayos comparativos directos de tofacitinib contra terapia convencional o biológica, en una revisión sistemática y metaanálisis en red de 17 ensayos realizada por Singh, et al. ${ }^{32}$ se comparó la 


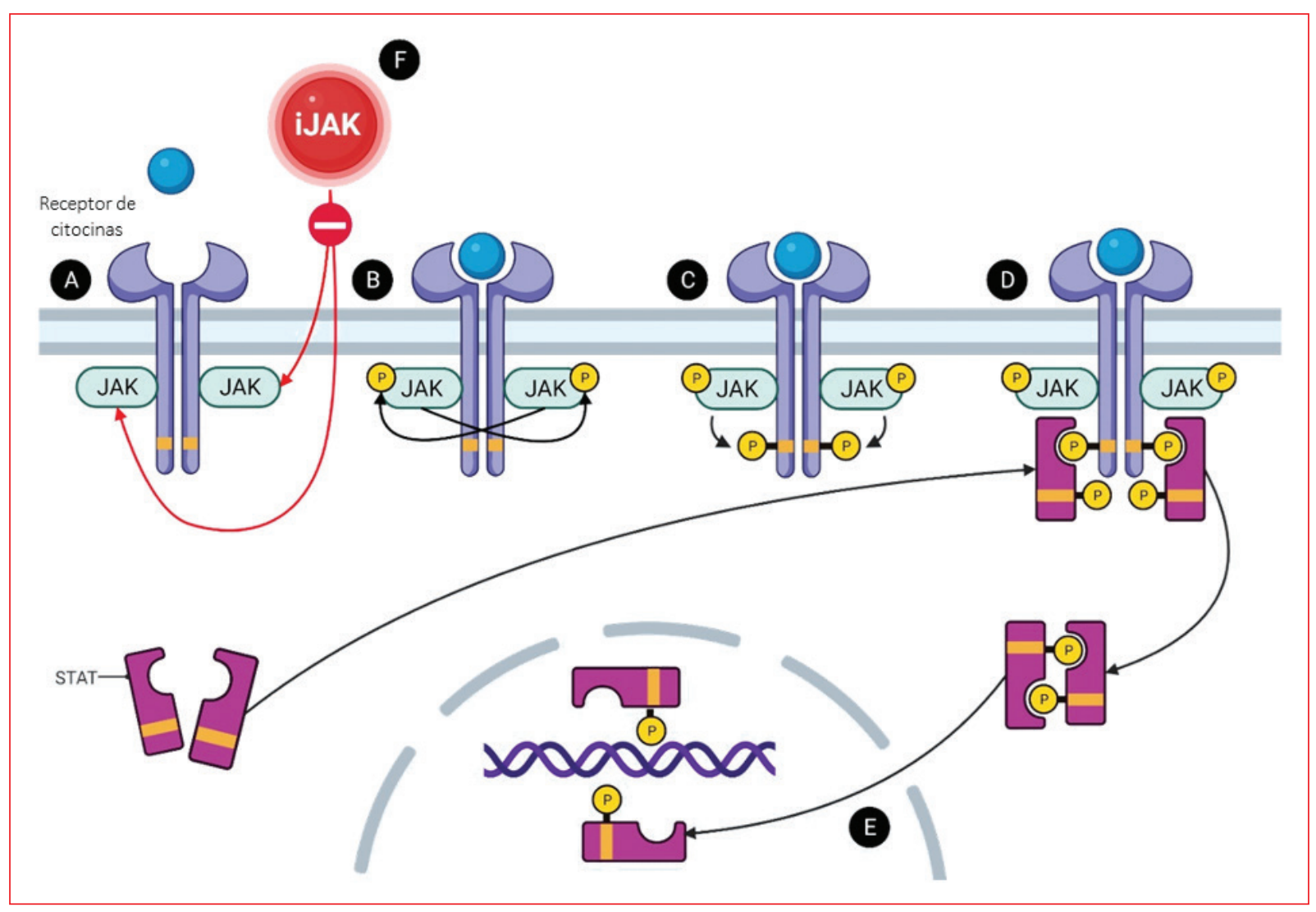

Figura 1. Vía JAK-STAT y su inhibición A: las JAK y los STAT están inactivos antes de la unión de un ligando. B y C: cuando una citocina se acopla a su receptor, las JAK se activan y se fosforilan entre sí, así como la porción intracelular de sus receptores. C: esto crea un sitio de acoplamiento para STAT, que permite unirse al dominio citoplasmático del receptor. D: las STAT, a su vez, se fosforilan y activan, lo que les permite dimerizarse. E: el dímero STAT-STAT se transloca al núcleo, donde puede unirse directamente al ADN y regular la expresión génica. F: los iJAK inhiben la fosforilación de JAK necesaria para el acoplamiento de STAT, impidiendo así su traslocación hacia el núcleo y la transcripción de proteínas inflamatorias. ADN: ácido desoxirribonucleico; iJAK: inhibidor de JAK; JAK: Janus cinasas; STAT: transductor de señal y activador de proteínas de transcripción.

eficacia del adalimumab, el vedolizumab, el tofacitinib y el ustekinumab como terapia de segunda línea (exposición previa a anti-TNF) para el tratamiento de la CU activa de moderada a grave, y se encontró que el tofacitinib (surface under the cumulative ranking curve: remisión clínica 0.87, mejoría endoscópica 0.91) y el ustekinumab (SUCRA: remisión clínica 0.87, mejoría endoscópica 0.83) lograron la puntuación más alta en cuanto a inducción de remisión clínica y mejoría endoscópica, superando al vedolizumab (tofacitinib frente a vedolizumab: odds ratio [OR] 6.18, intervalo de confianza del 95\% [IC95\%] 1.003-8.00; y ustekinumab frente a vedolizumab: OR 5.99, IC95\% 1.13-31.76) y al adalimumab (tofacitinib frente a adalimumab: OR 11.05, IC95\% 1.79-68.41; y ustekinumab frente a adalimumab: OR 10.71, IC95\% 2.01-57.20). Sin embargo, los resultados de esta revisión sistemática y metaanálisis en red deben tomarse con cautela, dado que ha recibido críticas por el alto riesgo de sesgo en los resultados debido al efecto de arrastre, no considerado durante el diseño metodológico ${ }^{33}$.

\section{Farmacocinética y farmacodinamia del tofacitinib}

La absorción del tofacitinib es rápida y las concentraciones plasmáticas máximas se alcanzan entre 0.5 y $1 \mathrm{~h}$ después de la administración ${ }^{34}$, mientras que su vida media funcional es de aproximadamente $3 \mathrm{~h}$ en sujetos sanos y en pacientes con $\mathrm{CU}^{34,35}$. El tofacitinib es metabolizado por el hígado a través del citocromo P450 (CYP3A4 y CYP2C19) ${ }^{36}$. Los metabolitos producidos tras su paso por el hígado no son clínicamente significativos, ya que su potencia para inhibir JAK1/ 
Tabla 1. Consideraciones clínicas y paraclínicas a tomar en cuenta antes y durante el tratamiento con tofacitinib

Condición

Edad $>65$ años

\section{Embarazo y lactancia}

Infección seria

Alto riesgo de infección:

- Infecciones recurrentes

- Antecedente de infección seria u oportunista

- Reside o ha viajado a áreas endémicas de micosis

- Tiene condiciones subyacentes que lo predisponen a una infección (diabetes, VIH/sida, enfermedad pulmonar intersticial, sistema inmunitario débil)

Tuberculosis

Latente 0 activa

Antecedente de tuberculosis

Reside o ha viajado a áreas endémica de tuberculosis

Serología viral VHB

VHC

$$
\begin{aligned}
& \text { Linfocitos } \\
& \text { absolutos } \\
& \text { (cel/mm } \left./ \mathrm{mm}^{3}\right)
\end{aligned}
$$

\begin{tabular}{|l|l|}
\hline & $500-750$ \\
\hline & $<500$ \\
\hline $\begin{array}{l}\text { Neutrófilos } \\
\text { absolutos } \\
\text { (cel/mm } 3 \text { ) }\end{array}$ & $>1000$ \\
\hline & $500-1000$ \\
\hline & $<500$ \\
\hline
\end{tabular}

\section{Recomendación}

- Usar si no existe tratamiento alternativo adecuado

- Usar con precaución

- No requiere ajuste de dosis

- No usar

- No usar

- Interrumpir hasta que la infección se controle

- Considerar los riesgos y beneficios antes de iniciar tofacitinib

- No usar

- Tratar con terapia antimicobacteriana estándar antes de considerar administrar tofacitinib

- Considerar los riesgos y beneficios antes de iniciar tofacitinib

- Realizar cribado antes de usar tofacitinib

- Los pacientes con antígeno de superficie de VHB (+) que reciben tofacitinib tienen una alta tasa de reactivación del VHB, que podría prevenirse mediante profilaxis antiviral ${ }^{28}$

- Los pacientes con antígeno de superficie de VHB (-) y anticuerpos anticore $(+)$ que reciben tofacitinib tienen un bajo riesgo de reactivación del VHB; se sugiere una estrecha monitorización del ADN del VHB y de la alanina aminotransferasa ${ }^{28,29}$

- Realizar cribado antes de usar tofacitinib

- Se desconoce el impacto del tofacitinib sobre el VHC, pero los resultados de un pequeño estudio no mostraron incremento de la replicación del $\mathrm{VHC}^{29}$

- Iniciar y vigilar a las 4-8 semanas, después cada 3 meses

- Mantener la dosis

- No iniciar

- Pacientes en tratamiento con tofacitinib $10 \mathrm{mg} \mathrm{c} / 12 \mathrm{~h}$ reducir a $5 \mathrm{mg} \mathrm{c} / 12 \mathrm{~h}$

- Pacientes en tratamiento con tofacitinib $5 \mathrm{mg} \mathrm{c} / 12 \mathrm{~h}$ interrumpir la dosificación

- Interrumpir si se corroboran cifras en laboratorios realizados en máximo 7 días

- Iniciar y vigilar a las 4-8 semanas, después cada 3 meses Mantener la dosis

- No usar

- Pacientes con tofacitinib $10 \mathrm{mg} \mathrm{c} / 12 \mathrm{~h}$ reducir a $5 \mathrm{mg} \mathrm{c} / 12 \mathrm{~h}$

- Pacientes con tofacitinib $5 \mathrm{mg} \mathrm{c} / 12 \mathrm{~h}$ interrumpir la dosificación

- Interrumpir si se corroboran cifras en laboratorios realizados en máximo 7 días 
Rev Mex Enferm Inflam Inmunomed. 2021;95(4)

Tabla 1. Consideraciones clínicas y paraclínicas a tomar en cuenta antes y durante el tratamiento con tofacitinib (continuación)

\begin{tabular}{|c|c|c|}
\hline \multicolumn{2}{|l|}{ Condición } & \multirow{2}{*}{$\begin{array}{l}\text { Recomendación } \\
\text { - Iniciar y vigilar a las 4-8 semanas, después cada } 3 \text { meses }\end{array}$} \\
\hline Hemoglobina (g/dl) & $<9$ & \\
\hline & $\geq 9$ & - No iniciar \\
\hline & Disminución de $\leq 2 \mathrm{y} \geq 9$ & - Mantener la dosis \\
\hline & Disminución de $\geq 2 \mathrm{y} \leq 8$ & - Interrumpir hasta que los valores se normalicen \\
\hline \multicolumn{2}{|l|}{ Lípidos } & $\begin{array}{l}\text { - Valorar a las } 8 \text { semanas de haber iniciado tofacitinib } \\
\text { - En caso de desarrollar hiperlipidemia, apegarse a las guías } \\
\text { clínicas para su manejo }\end{array}$ \\
\hline Enzimas hepáticas & Incremento de $>3$ veces LSN & - Reducir dosis o interrumpir tofacitinib \\
\hline \multirow[t]{3}{*}{ Disfunción hepática } & Child Pugh A & - No requiere ajuste \\
\hline & Child Pugh B & $\begin{array}{l}\text { - } 5 \mathrm{mg} \mathrm{c} / 24 \mathrm{~h} \text { cuando la dosis indicada en el paciente por lo demás } \\
\text { sano es } 5 \mathrm{mg} \mathrm{c} / 12 \mathrm{~h} \\
\text { - } 5 \mathrm{mg} \mathrm{c} / 12 \mathrm{~h} \text { cuando la dosis indicada en el paciente por lo demás } \\
\text { sano es } 10 \mathrm{mg} \mathrm{c} / 12 \mathrm{~h}\end{array}$ \\
\hline & Child Pugh C & - No usar \\
\hline \multirow[t]{3}{*}{ Disfunción renal } & ACr: $50-80 \mathrm{ml} / \mathrm{min}$ & - No requiere ajuste \\
\hline & ACr: $30-49 \mathrm{ml} / \mathrm{min}$ & - No requiere ajuste \\
\hline & ACr: $<30 \mathrm{ml} / \mathrm{min}$ o en hemodiálisis & $\begin{array}{l}\text { - } 5 \mathrm{mg} \mathrm{c} / 24 \mathrm{~h} \text { cuando la dosis indicada en el paciente por lo demás } \\
\text { sano es } 5 \mathrm{mg} \mathrm{c} / 12 \mathrm{~h} \text {. } \\
\text { - } 5 \mathrm{mg} \mathrm{c} / 12 \mathrm{~h} \text { cuando la dosis indicada en el paciente por lo demás } \\
\text { sano es } 10 \mathrm{mg} \mathrm{c} / 12 \mathrm{~h}\end{array}$ \\
\hline \multicolumn{2}{|c|}{$\begin{array}{l}\text { Uso concomitante: } \\
\text { - Anti-TNF } \\
\text { - Anti-IL12/23 } \\
\text { - Antiintegrinas } \\
\text { - Inmunomoduladores: azatioprina, 6-mercaptopurina, } \\
\text { ciclosporina y tacrolimus }\end{array}$} & - Evitar combinación \\
\hline \multirow[t]{3}{*}{ Interacciones } & $\begin{array}{l}\text { Inhibidor fuerte de CYP3A4 (p. ej., } \\
\text { ketoconazol) }\end{array}$ & \multirow[t]{2}{*}{$\begin{array}{l}\text { - Si está tomando } 10 \mathrm{mg} \mathrm{c} / 12 \mathrm{~h} \text {, reducir a } 5 \mathrm{mg} \mathrm{c} / 12 \mathrm{~h} \\
\text { - Si está tomando } 5 \mathrm{mg} \mathrm{c} / 12 \mathrm{~h} \text {, reducir a } 5 \mathrm{mg} \mathrm{c} / 24 \mathrm{~h}\end{array}$} \\
\hline & $\begin{array}{l}\text { Inhibidor moderado de CYP3A4 con } \\
\text { inhibición fuerte de CYP2C19 (p. ej., } \\
\text { fluconazol) }\end{array}$ & \\
\hline & $\begin{array}{l}\text { Inductor fuerte de CYP (p. ej., } \\
\text { rifampicina) }\end{array}$ & - Puede disminuir la eficacia del tofacitinib \\
\hline \multicolumn{2}{|l|}{ Vacunas } & $\begin{array}{l}\text { - Antes de iniciar tofacitinib hay que asegurar que el esquema de } \\
\text { vacunación se encuentre actualizado de acuerdo con las pautas } \\
\text { vigentes } \\
\text { - No administrar vacunas vivas al mismo tiempo que tofacitinib } \\
\text { - La vacuna viva debe aplicarse al menos } 2 \text { semanas, pero } \\
\text { preferiblemente } 4 \text { semanas, antes del inicio de tofacitinib }\end{array}$ \\
\hline \multicolumn{2}{|c|}{$\begin{array}{l}\text { Presencia de factores de riesgo para TEV: } \\
\text { - TVE previa } \\
\text { - Paciente sometido a cirugía mayor } \\
\text { - Inmovilización } \\
\text { - Infarto al miocardio en } 3 \text { meses previos } \\
\text { - Falla cardiaca } \\
\text { - Uso de anticonceptivos hormonales combinados o terapia } \\
\text { de reemplazo hormonal } \\
\text { - Trastorno de coagulación hereditario } \\
\text { - Neoplasia } \\
\text { - IMC } \geq 30 \\
\text { - Diabetes mellitus } \\
\text { - Hipertensión } \\
\text { - Tabaquismo }\end{array}$} & $\begin{array}{l}\text { - Usar si no existe tratamiento alternativo adecuado } \\
\text { - Usar con precaución } \\
\text { - Considerar medir los niveles de dímero D después de } 12 \text { meses } \\
\text { de tratamiento: si } \geq 2 \text { veces LSN, confirmar que los beneficios } \\
\text { clínicos superan los riesgos antes de tomar una decisión sobre } \\
\text { la continuación del tratamiento con tofacitinib }\end{array}$ \\
\hline
\end{tabular}


Tabla 1. Consideraciones clínicas y paraclínicas a tomar en cuenta antes y durante el tratamiento con tofacitinib (continuación)

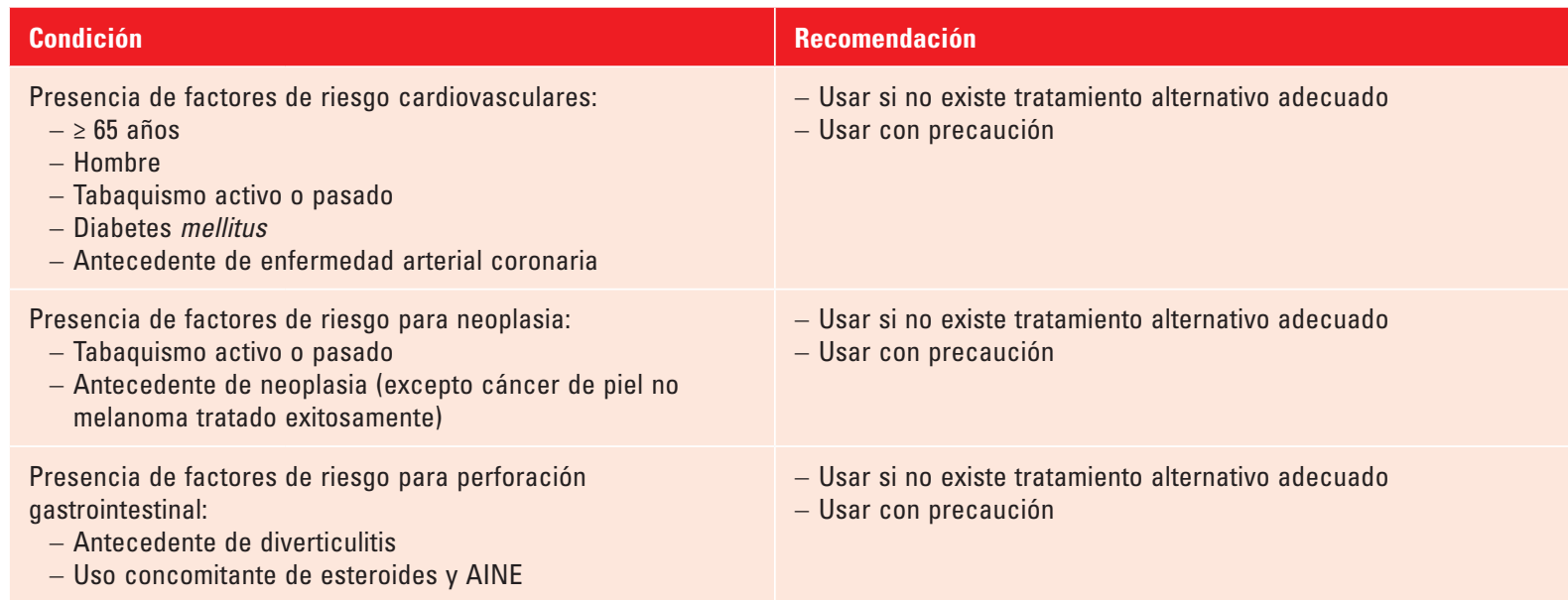

ACr: aclaramiento de creatinina; AINE: antinflamatorios no esteroideos; IL: interleucina; IMC: índice de masa corporal; LSN: límite superior de la normalidad; TEV: tromboembolia venosa; TNF: factor de necrosis tumoral; VHB: virus de la hepatitis B; VHC: virus de la hepatitis C; VIH: virus de la inmunodeficiencia humana. Fuente: elaboración propia a partir de datos de refs. $4-6,28$ y 29.

JAK3 es $\leq 10 \%$ de la observada para el tofacitinib. Aproximadamente el $80 \%$ y el $20 \%$ de tofacitinib y de los metabolitos relacionados se eliminan en la orina y las heces, respectivamente, en sujetos sanos ${ }^{34}$.

El tofacitinib es un potente paninhibidor de la familia JAK (JAK1, JAK2, JAK3 y TyK2). En las células humanas, inhibe preferentemente la señalización de los receptores de citocinas que se asocian con JAK3 o JAK1. La inhibición de JAK1 y JAK3 por el tofacitinib atenúa la señalización de las interleucinas (IL) 2, 4, 6, $7,9,15$ y 21) y de los interferones tipo I y tipo II, lo que da como resultado la modulación del sistema inmunitario y de la respuesta inflamatoria.

\section{Posología del tofacitinib}

La dosis de inducción recomendada es de $10 \mathrm{mg}$ por vía oral, con o sin alimentos, dos veces al día durante 8 semanas. Para los pacientes que no logran un beneficio terapéutico adecuado a la semana 8 , la dosis de inducción de $10 \mathrm{mg}$ dos veces al día se puede extender por otras 8 semanas (16 semanas en total). La terapia de inducción con tofacitinib debe suspenderse en cualquier paciente que no muestre evidencia de beneficio terapéutico en la semana $16^{19,20,37}$.

La dosis de mantenimiento recomendada de tofacitinib es de $5 \mathrm{mg}$ por vía oral dos veces al día. Para los pacientes con $\mathrm{CU}$ que no tienen mayor riesgo de tromboembolia venosa se pueden considerar $10 \mathrm{mg}$ por vía oral dos veces al día si el paciente experimenta una pobre respuesta con $5 \mathrm{mg}$ dos veces al día y no responde a opciones de tratamiento alternativas para la
CU, como el tratamiento con anti-TNF. Se debe utilizar tofacitinib $10 \mathrm{mg}$ dos veces al día para el tratamiento de mantenimiento durante el menor tiempo posible, teniendo en cuenta siempre utilizar la dosis eficaz más baja necesaria para mantener la respuesta ${ }^{19,20}$.

Antes de iniciar o durante el tratamiento existen ciertas consideraciones clínicas y paraclínicas que deben ser tomadas en cuenta, dado que inciden en la decisión de iniciar, ajustar o retirar el tofacitinib de acuerdo con las características del paciente ${ }^{19,20,36,38-40}$ (Tabla 2).

\section{Valoración de la respuesta}

El conocimiento de los objetivos clínicos, bioquímicos y endoscópicos es de vital importancia para mejorar el manejo de los pacientes con CU. El grupo STRIDE II recomienda que posterior al inicio del tratamiento con tofacitinib se valore la respuesta clínica en 6 semanas, la remisión clínica en 11 semanas, la normalización de la proteína $C$ reactiva o la velocidad de sedimentación globular en 9 semanas, la disminución de la calprotectina fecal por debajo del umbral en 11 semanas y la cicatrización de la mucosa en 14 semanas.

\section{Eventos adversos y seguridad}

En general, el tofacitinib ha mostrado un buen perfil de seguridad en los pacientes con CU. Sin embargo, debido a los amplios efectos inmunosupresores por la inhibición de JAK, es primordial definir la relación entre riesgo y beneficio de su empleo en la Ell, pues esto influirá en los patrones de su uso ${ }^{3-5}$. 
Tabla 2. Nuevos fármacos inhibidores de JAK en desarrollo para el tratamiento de la colitis ulcerosa

\begin{tabular}{|l|l|l|l|l|}
\hline Compuesto & Diana terapéutica & Ensayo clínico & $\begin{array}{l}\text { Final estimado del } \\
\text { ensayo }\end{array}$ & Patrocinador \\
\hline Filgotinib (GLPG0634) & JAK-1 & NCT02914522: fase 3 & $\begin{array}{l}\text { Marzo 2020 } \\
\text { Diciembre 2023 }\end{array}$ & Gilead Sciences \\
\hline $\begin{array}{l}\text { Upadacitinib } \\
\text { (ABT-494) }\end{array}$ & JAK1 & NCT02819635: fase 3 & Enero 2022 & AbbVie \\
\hline $\begin{array}{l}\text { Izencitinib } \\
\text { (D-1473) }\end{array}$ & $\begin{array}{l}\text { Paninhibidor con } \\
\text { acción restringida al } \\
\text { intestino }\end{array}$ & $\begin{array}{l}\text { NCT02818686: fase 1 } \\
\text { NCT03758443: fase 2-3 } \\
\text { NCT03920254: fase 2-3 }\end{array}$ & $\begin{array}{l}\text { Marzo 2018 } \\
\text { Julio 2025 } \\
\text { Septiembre 2028 }\end{array}$ & $\begin{array}{l}\text { Theravance } \\
\text { Biopharma }\end{array}$ \\
\hline $\begin{array}{l}\text { Ritlecitinib } \\
\text { (Pf-06651600) } \\
\text { Brepocitinib } \\
\text { (Pf-0670084) }\end{array}$ & $\begin{array}{l}\text { JAK-3 } \\
\text { JAK1 / TYK2 }\end{array}$ & NCT02958865: fase 2 & Mayo 2021 & Pfizer \\
\hline \begin{tabular}{l} 
Deucravacitinib (BMS-986165) \\
\hline
\end{tabular} & TYK2 & $\begin{array}{l}\text { NCT04613518: fase 2 } \\
\text { NCT03934216: fase 2 } \\
\text { NCT04877990: fase 2 }\end{array}$ & $\begin{array}{l}\text { Abril 2023 } \\
\text { Abril 2023 } \\
\text { Junio 2027 }\end{array}$ & Bristol-Myers Squibb \\
\hline
\end{tabular}

Fuente: elaboración propia a partir de datos de refs. 30-39.

Una revisión sistemática y metaanálisis realizado recientemente muestra que la frecuencia de eventos adversos y de eventos adversos serios es similar entre los expuestos a tofacitinib (5 y $10 \mathrm{mg}$ ) y placebo ${ }^{41}$.

Eventos adversos como infecciones serias, infecciones por virus herpes zóster, neoplasias malignas, cáncer de piel no melanoma, tromboembolia y eventos cardiovasculares mayores han recibido especial interés ${ }^{20}$.

\section{INFECCIONES}

En los ensayos OCTAVE, las infecciones tuvieron tasas más altas con tofacitinib en comparación con placebo. La tasa de infección grave fue mayor con tofacitinib en los ensayos de inducción, pero similar en los grupos de tratamiento en el ensayo de mantenimiento ${ }^{18}$.

En una revisión sistemática y metaanálisis realizado por Wang, et al. ${ }^{41}$ en el año 2020 se encontró que el riesgo de infección aumentó significativamente solo con tofacitinib $10 \mathrm{mg}$ dos veces al día (riesgo relativo: 2.75; IC95\%: 1.72-4.41; $p<0,001)$. En cuanto a las infecciones graves (excluyendo el herpes zóster), la incidencia general fue similar a la del placebo ${ }^{41}$.

\section{INFECCIÓN POR VIRUS HERPES ZÓSTER}

Los ensayos OCTAVE mostraron una tasa más alta de infección por virus herpes zóster en el grupo de $10 \mathrm{mg}$ de tofacitinib en comparación con el grupo de placebo y con el grupo de $5 \mathrm{mg}$ de tofacitinib ${ }^{18}$. Sin embargo, en revisiones sistemáticas y metaanálisis realizados recientemente no se observó un riesgo estadísticamente mayor de infección por virus herpes zóster con tofacitinib 5 y $10 \mathrm{mg}^{41,42}$.

\section{TuberCulosis}

El riesgo de tuberculosis en los pacientes con Ell es bajo y depende de la carga local de tuberculosis, siendo la prevalencia del $0.02 \%$, el $0.21 \%$ y el $1.59 \%$ en los países con carga de tuberculosis baja, intermedia y alta, respectivamente ${ }^{43}$. Sin embargo, los pacientes deben ser evaluados para detectar tuberculosis latente y activa antes de recibir la terapia con tofacitinib, e iniciar medicamentos antituberculosos en aquellos con tuberculosis latente ${ }^{44}$.

\section{CÁncer de piel no melanoma y otras NEOPLASIAS}

Según metaanálisis recientes, el tofacitinib no parece aumentar el riesgo de cáncer de piel no melanoma y otras neoplasias malignas ${ }^{42,45,46}$. Sin embargo, persiste la preocupación por el riesgo de desarrollar neoplasias malignas y cánceres de piel no melanoma especialmente en pacientes tratados con tofacitinib $10 \mathrm{mg}$ dos veces al día. Por lo tanto, se recomienda una vigilancia estrecha para cualquier neoplasia maligna y el examen periódico de la piel para pacientes que tienen un mayor riesgo de cáncer de piel${ }^{20}$. 


\section{TROMBOEMBOLIA}

En los estudios OCTAVE de inducción y mantenimiento se produjeron episodios de trombosis venosa profunda (TVP) o embolia pulmonar (EP) en cinco pacientes (una TVP y cuatro EP), todos ellos tratados con tofacitinib $10 \mathrm{mg}$ dos veces al día ${ }^{18}$. Una revisión sistemática de siete estudios no demostró un mayor riesgo de TVP o EP en pacientes expuestos a tofacitinib en comparación con placebo ${ }^{42}$. Sin embargo, existen factores de riesgo para desarrollar TVP o EP que deben ser considerados antes del inicio del tratamiento 20,36 (Tabla 2).

\section{Eventos CARDIOVASCULARES MAYORES}

La inhibición de JAK se ha asociado con alteraciones del perfil de lípidos séricos y la posible aparición de eventos cardiovasculares mayores ${ }^{20,36}$. Sin embargo, los cambios observados en los niveles de colesterol son pequeños y transitorios ${ }^{47}$. Un metaanálisis de 17 estudios no mostró un riesgo significativamente mayor de padecer eventos cardiovasculares mayores en aquellos pacientes expuestos a tofacitinib en comparación con placebo ${ }^{42}$.

\section{Otros eVEnTOS ADVERSOS}

Otros eventos adversos posibles descritos en la literatura son perforación gastrointestinal, alteraciones de laboratorio caracterizadas por un incremento de los parámetros lipídicos y de las enzimas hepáticas, y un decremento de las cifras de hemoglobina, linfocitos y neutrófilos ${ }^{20,36,48}$. Se recomienda, previo y durante el tratamiento, valorar el comportamiento de estos parámetros y la presencia o no de factores de riesgo para el desarrollo de perforación gastrointestinal ${ }^{20,36}$ (Tabla 2).

\section{Poblaciones especiales}

La seguridad del tofacitinib en niños, mujeres embarazadas y pacientes de edad avanzada no se ha estudiado directamente en la CU. Sin embargo, existe evidencia limitada obtenida del uso de tofacitinib en estas poblaciones especiales para el tratamiento de otras enfermedades diferentes de la $\mathrm{CU}^{49}$.

\section{NiÑos}

No se han establecido la seguridad y la eficacia del tofacitinib en menores de 18 años con $\mathrm{CU}^{20}$.
Actualmente, el tofacitinib solo se encuentra autorizado por la FDA y la EMA para niños > 2 años con artritis idiopática juvenil poliarticular y artritis psoriásica juvenil ${ }^{20,36}$. Un estudio retrospectivo incluyó 21 sujetos con Ell (18 de ellos con CU) de 21 años o menos que recibieron tofacitinib para la Ell médicamente refractaria. Al final del periodo de inducción de 12 semanas, el $42.9 \%$ mostraron respuesta clínica y el $33.3 \%$ estaban en remisión libre de esteroides. De los sujetos evaluables a las 52 semanas, el $41.2 \%$ mostraron respuesta clínica y estaban en remisión libre de esteroides. De los que siguieron con tofacitinib al año, ninguno requirió corticosteroides sistémicos concomitantes. Se suspendió el tofacitinib en ocho sujetos debido a enfermedad refractaria, incluidos ocho que finalmente se sometieron a colectomía y uno que desarrolló un absceso intraabdominal estéril. No hubo casos de trombosis, reactivación del herpes zóster 0 hiperlipidemia clínicamente significativa ${ }^{50}$.

\section{Adultos mayores}

En pacientes de 65 años o más, debido a un mayor riesgo de infecciones, infarto al miocardio, trombosis y cáncer, el tofacitinib solo debe considerarse si no se dispone de un tratamiento alternativo adecuado 20,36,51. No es necesario ajustar la dosis en pacientes de 65 años o mayores 20,36 .

\section{FERTILIDAD, EMBARAZO Y LACTANCIA}

El uso de tofacitinib durante el embarazo y la lactancia está contraindicado ${ }^{20,51}$.

Aunque no hay datos sobre la transferencia placentaria del tofacitinib, es razonable suponer que este fármaco, una molécula de pequeño tamaño, atraviesa la placenta desde el comienzo del embarazo ${ }^{52}$.

En estudios con animales, el tofacitinib fue feticida y teratogénico en ratas y conejos, aunque a exposiciones muchas veces mayores que la dosis estándar en humanos. Los resultados notificados de los casos de embarazo identificados a partir de los ensayos controlados aleatorizados de tofacitinib, los estudios de no intervención posteriores a la aprobación y la notificación espontánea de eventos adversos, parecen similares a los observados en la población general. Sin embargo, en la actualidad, debe evitarse el uso de tofacitinib durante el embarazo ${ }^{53}$. Se deben utilizar métodos anticonceptivos 
Tabla 3. Esquema de vacunación recomendado en pacientes bajo tratamiento inmunosupresor

\section{Vacuna}

VII tetravalente de dosis estándar

VII de dosis alta o vacuna contra la influenza adyuvada

Vacuna contra la influenza viva atenuada

Vacuna contra el herpes zóster recombinante

Vacuna 9-valente contra el virus del papiloma humano

PCV-13 y PPSV-23

Hepatitis A

Hepatitis B

Vacuna contra la varicela viva atenuada

SRP viva atenuada

\section{Programa de dosificación}

1 dosis estacionalmente para todos los pacientes de 18-64 años que no reciben monoterapia anti-TNF- $\alpha$

1 dosis estacionalmente para pacientes $\geq 65$ años o 18-64 años si reciben monoterapia anti-TNF- $\alpha$

Evitar en pacientes con agentes inmunosupresores

2 dosis (con 2-6 meses de diferencia) para todos los pacientes $\geq 50$ años

Considerar para pacientes de 40-49 años con mayores factores de riesgo de herpes zóster:

- Antecedente de herpes zóster

- Requerir cursos repetidos de corticosteroides

- Tomando tofacitinib con factores de riesgo (uso concurrente de corticosteroides, asiáticos, diabetes mellitus, falla previa de anti-TNF o uso prolongado de $10 \mathrm{mg}$ de tofacitinib dos veces al día)

- En terapia combinada y que requieren corticosteroides

Todos los pacientes, de ambos sexos, de 18-26 años con Ell deben vacunarse con un régimen de 3 dosis (a los $0,1-2$ y 6 meses)

Serie de 3 dosis para pacientes de 27-45 años si es probable que tengan nuevas parejas sexuales

Todas las personas $\geq 18$ años

Si no hay vacunación previa, PCV13 seguida de 1 dosis de PPSV23 después de $\geq 8$ semanas; si recibió $\geq 1$ dosis de PPSV23, debería recibir PCV13 $\geq 1$ año después de PPSV23

Se debe administrar otra dosis de PPSV23 5 años después de la dosis inicial de PPSV23 y a la edad $\geq 65$ años si han transcurrido $\geq 5$ años desde la dosis anterior de PPSV23

Verificar el estado inmunitario para la hepatitis A en la visita inicial del paciente; si no es inmune, vacunar con uno de los siguientes esquemas:

- Serie de 2 dosis de Hep A: Havrix ${ }^{\circledR}$ con 6-12 meses de diferencia o Vaqta ${ }^{\circledR}$ con 6-18 meses de diferencia; intervalo mínimo: 6 meses

- Serie de 3 dosis de Hep A-Hep B: Twinrix ${ }^{\circledR}$ a los 0, 1, 6 meses

- Programa de dosificación acelerada de 4 dosis a los 0, 7, 21-30 días y 12 meses (destinado a pacientes que comienzan la serie de vacunación, pero no pueden completar el programa estándar de 3 dosis debido a un viaje de alto riesgo anticipado)

Verificar el estado inmunitario para la hepatitis B en la visita inicial del paciente; si no es inmune, vacunar con uno de los siguientes esquemas:

- Serie de 3 dosis de Engerix- $B^{\circledR}$ o Recombivax ${ }^{\circledR} H B$ a los 0, 1, 6 meses

- Serie de 3 dosis de Hep A-Hep B a los 0, 1,6 meses

- Serie de 2 dosis de HepB-CpG (Heplisav- ${ }^{\circledR}$ ) a los 0 y 1 mes

Verificar los anticuerpos contra el antígeno de superficie 4-8 semanas después de completar la serie

Vacunar a todos los pacientes no inmunes siempre que no hayan recibido terapia inmunosupresora sistémica en los 3 meses anteriores y no haya planes para comenzar la terapia inmunosupresora en las próximas 6 semanas

Serie de 2 dosis con un intervalo de 4-8 semanas si anteriormente no recibió la vacuna contra la varicela

Vacunar a todos los pacientes no inmunes siempre que no hayan recibido agentes inmunosupresores sistémicos en los 3 meses anteriores y no haya planes para comenzar con agentes inmunosupresores en las próximas 6 semanas

Serie de 2 dosis con al menos 4 semanas de diferencia si anteriormente no recibió ninguna SRP 0 1 dosis si recibió previamente 1 dosis de SRP

Ell: enfermedad inflamatoria intestinal; PCV-13: vacuna conjugada antineumocócica 13-valente; PPSV-23: vacuna antineumocócica de polisacáridos 23-valente; SRP: sarampión, rubeola y parotiditis; TNF: factor de necrosis tumoral; VII: vacuna contra la influenza inactiva. Fuente: traducida de ref. 17.

Aunque ningún estudio en humanos ha informado de resultados sobre la lactancia materna de madres expuestas a tofacitinib, este fármaco está presente en la leche de rata, por

lo que en la actualidad se debe evitar la lactancia materna ${ }^{53}$. eficaces durante el tratamiento y durante 4-6 semanas después de la última dosis de tofacitinib ${ }^{15}$. El embarazo en pacientes con exposición paterna a tofacitinib parece ser seguro ${ }^{53}$. 


\section{Vacunación}

Los pacientes con Ell tienen un mayor riesgo de infecciones graves y de herpes zóster, y este riesgo aumenta con el tratamiento con tofacitinib y terapias combinadas $^{19}$. Por ello, parece aconsejable, como medida preventiva, mantener al día el esquema de vacunación antes de iniciar el tratamiento con tofacitinib de acuerdo con las guías de vacunación en los pacientes con Ell bajo inmunosupresión ${ }^{20,36,54}$ (Tabla 3). Es importante señalar que la vacunación con vacunas vivas debe realizarse al menos 2 semanas (preferiblemente 4 semanas) antes del inicio del tofacitinib ${ }^{20}$.

\section{Diferencias entre los inhibidores de JAK y otras terapias biológicas}

Durante muchos años, la CU se ha tratado principalmente con corticosteroides, aminosalicilatos, inmunosupresores e inhibidores del TNF. Sin embargo, el desarrollo reciente de fármacos y productos biológicos de molécula pequeña ha mejorado el tratamiento y, por tanto, el pronóstico de los pacientes con Ell48.

Los iJAK tienen muchas ventajas en comparación con la terapia biológica, como son menores costos de producción, no requieren ajuste de la dosis al peso, fácil administración oral e independiente de los alimentos, rápido inicio de sus efectos, vida media corta de 3 horas, no inmunogénicos debido a su pequeño tamaño molecular y por lo tanto con posibilidad de utilizar el fármaco de forma intermitente sin riesgo de generar anticuerpos contra él ${ }^{15,20,48,51,55}$.

\section{Financiamiento}

La presente investigación es sin ánimo de lucro y no ha recibido ninguna beca de agencias de los sectores público o privado.

\section{Conflicto de intereses}

Los autores declaran no tener conflicto de intereses.

\section{Responsabilidades éticas}

Protección de personas y animales. Los autores declaran que para esta investigación no se han realizado experimentos en seres humanos ni en animales.
Confidencialidad de los datos. Los autores declaran que en este artículo no aparecen datos de pacientes.

Derecho a la privacidad y consentimiento informado. Los autores declaran que en este artículo no aparecen datos de pacientes.

\section{Referencias}

1. Turner D, Ricciuto A, Lewis A, D'Amico F, Dhaliwal J, Griffiths A, et al. International Organization for the Study of IBD. STRIDE-II: an update on the Selecting Therapeutic Targets in Inflammatory Bowel Disease (STRIDE) Initiative of the International Organization for the Study of IBD (IOIBD): determining therapeutic goals for treat-to-target strategies in IBD. Gastroenterology. 2021;160(5):1570-83.

2. Soendergaard C, Bergenheim F, Bjerrum J, Nielsen O. Targeting JAKSTAT signal transduction in IBD. Pharmacol Ther. 2018;192:100-11.

3. Salas A, Hernandez-Rocha C, Duijvestein M, Faubion W, McGovern D, Vermeire S, et al. JAK-STAT pathway targeting for the treatment of inflammatory bowel disease. Nat Rev Gastroenterol. 2020;17(6):323-37.

4. Hazel K, O'Connor A. Emerging treatments for inflammatory bowel disease. Ther Adv Chronic Dis. 2020;11:2040622319899297.

5. Aaronson D, Horvath C. A road map for those who don't know JAK-STAT. Science. 2002;296(5573):1653-5.

6. O'Shea J, Schwartz D, Villarino A, Gadina M, Mclnnes I, Laurence A The JAK-STAT pathway: impact on human disease and therapeutic intervention. Annu Rev Med. 2015;66:311-28.

7. Verstockt B, Ferrante M, Vermeire S, Van Assche G. New treatment options for inflammatory bowel diseases. J Gastroenterol. 2018;53(5):585-90.

8. Wilks AF. Two putative protein-tyrosine kinases identified by application of the polymerase chain reaction. Proc Natl Acad Sci. 1989;86(5):1603-7.

9. Wilks A, Harpurc A, Kurban R, Ralph S, Zürcher G, Ziemiecki A. Two novel protein-tyrosine kinases, each with a second phosphotransferase-related catalytic domain, define a new class of protein kinase. Mol Cell Biol. 1991;11(4):2057-65.

10. Johnston JKM, Kirken R, Chen Y, Blake T, Shibuya K, Ortaldo J, et al Phosphorylation and activation of the Jak-3 Janus kinase in response to interleukin-2. Nature. 1994; 370(6485):151-3.

11. Rane S, Reddy E. JAK3: a novel JAK kinase associated with terminal differentiation of hematopoietic cells. Oncogene. 1994;9(8):2415-23.

12. Liongue $C$, Taznin T, Ward A. Signaling via the CytoR/JAK/STAT/SOCS pathway: emergence during evolution. Mol Immunol. 2016;71:166-75.

13. Coskun M, Salem M, Pedersen J, Nielsen O. Involvement of JAK/STAT signaling in the pathogenesis of inflammatory bowel disease. Pharmacol Res. 2013;76:1-8.

14. Kisseleva T, Bhattacharya S, Braunstein J, Schindler C. Signaling through the JAK/STAT pathway, recent advances and future challenges. Gene. 2002; 285(1-2):1-24.

15. Roda G, Dal Buono A, Argollo M, Danese S. JAK selectivity: more precision less troubles. Expert Rev Gastroenterol Hepatol. 2020;14(9):789-96.

16. Fernández-Clotet A, Castro-Poceiro J, Panés J. JAK inhibition: the most promising agents in the IBD pipeline? Curr Pharm Des. 2019;25(1):32-40.

17. Agrawal M, Kim E, Colombel J. JAK inhibitors safety in ulcerative colitis: practical implications. J Crohns Colitis. 2020;14(Suppl 2):S755-60.

18. Sandborn W, Su C, Sands B, D'Haens G, Vermeire S, Schreiber S, et al. OCTAVE Induction 1, OCTAVE Induction 2, and OCTAVE Sustain Investigators. Tofacitinib as induction and maintenance therapy for ulcerative colitis. N Engl J Med. 2017; 376(18):1723-36.

19. U.S. Food \& Drug Administration. Xeljanz. 2018. Citado: 25/10/2021. Disponible en: https://www.accessdata.fda.gov/drugsatfda_docs/label/2018/203214s018lbl.pdf

20. European Medicines Agency. Xeljanz. 2021. Citado: 25/10/2021. Disponible en: https://www.ema.europa.eu/en/documents/product-information/ xeljanz-epar-product-information_en.pdf

21. National Institute of Health: U.S. National Library of Medicine. ClinicalTrials.gov. 2021. Citado: 27/10/2021. Disponible en: https://clinicaltrials. gov/ct2/show/NCT02914522?term=filgotinib\&draw=3\&rank=19

22. National Institutes of Health: U.S. National Library of Medicine. ClinicalTrials.gov. 2021. Citado: 25/10/2021. Disponible en: https://clinicaltrials. gov/ct2/show/study/NCT02914535?term=filgotinib\&draw=2\&rank=8

23. National Institute of Health: U.S. National Library of Medicine. ClinicalTrials.gov. 2021. Citado: 25/10/2021. Disponible en: https://clinicaltrials. gov/ct2/show/study/NCT02819635

24. National Institute of Health: U.S. National Library of Medicine. ClinicalTrials.gov. 2021. Citado: 25/10/2021. Disponible en: https://clinicaltrials. gov/ct2/show/NCT02818686?term $=$ TD $-1473 \&$ draw $=2 \&$ rank $=5$ 
Rev Mex Enferm Inflam Inmunomed. 2021;95(4)

25. National Institute of Health: U.S. National Library of Medicine. ClinicalTrials.gov. 2021. Citado: 25/10/2021. Disponible en: https://clinicaltrials. gov/ct2/show/NCT03758443?term $=$ TD-1473\&draw=2\&rank=6

26. National Institute of Health: U.S. National Library of Medicine. ClinicalTrials.gov. 2021. Citado: 25/10/2021. Disponible en: https://clinicaltrials gov/ct2/show/NCT03920254?term=TD-1473\&draw=2\&rank=2

27. Medicine NloHUSNLo. ClinicalTrials.gov. 2021. Citado: 25/10/2021. Disponible en: https://clinicaltrials.gov/ct2/show/NCT02958865?ter$m=P f-06651600$ \&draw $=4$ \&rank $=23$

28. National Institute of Health: U.S. National Library of Medicine. ClinicalTrials.gov. 2021. Citado: 27/10/2021. Disponible en: https://clinicaltrials. gov/ct2/show/NCT04613518?term=BMS-986165\&draw=4\&rank=25

29. National Institute of Health: U.S. National Library of Medicine. ClinicalTrials.gov. 2021. Citado: 27/10/2021. Disponible en: https://clinicaltrials. gov/ct2/show/NCT03934216?term=BMS-986165\&draw $=6 \&$ rank $=42$

30. National Institute of Health: U.S. National Library of Medicine. ClinicalTrials.gov. 2021. Citado: 27/10/2021. Disponible en: https://clinicaltrials. gov/ct2/show/NCT04877990?term=BMS-986165\&draw=2\&rank=2

31. Lefevre $P$, Vande Casteele N. Clinical pharmacology of Janus kinase inhibitors in inflammatory bowel disease. J Crohns Colitis. 2020; 14(Suppl 2):S725-36.

32. Singh SMM, Fumery M, Dulai $P$, Sandborn W. First- and second-line pharmacotherapies for patients with moderate to severely active ulcerative colitis: an updated network meta-analysis. Clin Gastroenterol Hepatol. 2020;18(10):2179-97.e6

33. Naessens D, Cameron C, Hoaglin D. Network meta-analysis of ulcerative colitis pharmacotherapies: carryover effects from induction and bias of the results. Clin Gastroenterol Hepatol. 2021;19(10):2219-21.

34. Dowty M, Lin J, Ryder T, Wang W, Walker G, Vaz A, et al. The pharmacokinetics, metabolism, and clearance mechanisms of tofacitinib, a Janus kinase inhibitor, in humans. Drug Metab Dispos. 2014;42(4):759-73.

35. Mukherjee A, Hazra A, Smith M, Martin S, Mould D, Su C, et al. Exposure-response characterization of tofacitinib efficacy in moderate to severe ulcerative colitis: Results from a dose-ranging phase 2 trial. $\mathrm{Br} \mathrm{J}$ Clin Pharmacol. 2018;84(6):1136-45.

36. Pfizer, Inc. Clinical pharmacology and biopharmaceutics review. 2011. Citado: 25/10/2021. Disponible en: https://www.accessdata.fda.gov/drugsatfda_docs/nda/2012/2032140rig1s000ClinPharmR.pdf

37. Feuerstein JD, Isaacs KL, Schneider Y, Siddique SM, Falck-Ytter $Y$, Singh S; AGA Institute Clinical Guidelines Committee. AGA Clinical practice guidelines on the management of moderate to severe ulcerative colitis. Gastroenterology. 2020;158(5):1450-61.

38. Wang S, Tseng C, Hsu C, Tung C, Huang K, Lu M, et al. Reactivation of hepatitis $B$ virus infection in patients with rheumatoid arthritis receiving tofacitinib. Int J Rheum Dis. 2021;24(11):1362-9.

39. Serling-Boyd N, Mohareb A, Kim A, Hyle E, Wallace Z. The use of tocilizumab and tofacitinib in patients with resolved hepatitis $B$ infection: a case series. Ann Rheum Dis. 2021;80(2):274-6.

40. Chen Y, Huang W, Liao T, Chen J, Yang S, Chen H, et al. Comparisons of hepatitis $\mathrm{C}$ viral replication in patients with rheumatoid arthritis receiving tocilizumab, abatacept and tofacitinib therapy. Ann Rheum Dis. 2019;78(6):849-50.
41. Wang F, Sun L, Wang S, Davis J, Matteson E, Murad M, et al. Efficacy and safety of tofacitinib, baricitinib, and upadacitinib for rheumatoid arthritis: a systematic review and meta-analysis. Mayo Clin Proc. 2020;95(7):1404-19.

42. Olivera P, Lasa J, Bonovas S, Danese S, Peyrin-Biroulet L. Safety of Janus kinase inhibitors in patients with inflammatory bowel diseases or other immune-mediated diseases: a systematic review and meta-analysis. Gastroenterology. 2020;158(6):1554-73.

43. Kedia S, Mouli V, Kamat N, Sankar J, Ananthakrishnan A, Makharia G, et al. Risk of tuberculosis in patients with inflammatory bowel disease on infliximab or adalimumab is dependent on the local disease burden of tuberculosis: a systematic review and meta-analysis. Am J Gastroenterol. 2020;115(3):340-9

44. Garrido I, Lopes S, Macedo G. Hit the road JAK! The role of new ora treatment in inflammatory bowel disease. Inflamm Bowel Dis. 2021 Mar 20;izab037. doi: 10.1093/ibd/izab037. Online ahead of print.

45. Maneiro J, Souto A, Gomez-Reino J. Risks of malignancies related to tofacitinib and biological drugs in rheumatoid arthritis: systematic review, meta-analysis, and network meta-analysis. Semin Arthritis Rheum. 2017;47(2):149-56

46. Xie W, Yang X, Huang $\mathrm{H}$, Gao D, Ji L, Zhang Z. Risk of malignancy with non-TNFi biologic or tofacitinib therapy in rheumatoid arthritis: a meta-analysis of observational studies. Semin Arthritis Rheum. 2020;50:930-

47. Wu J, Strober B, Hansen $P$, Ahlehoff O, Egeberg A, Qureshi A, et al. Effects of tofacitinib on cardiovascular risk factors and cardiovascular outcomes based on phase III and long-term extension data in patients with plaque psoriasis. J Am Acad Dermatol. 2016;75(5):897905.

48. Kim J, Kim S. The era of Janus kinase inhibitors for inflammatory bowel disease treatment. Int J Mol Sci. 2021;22(21):11322.

49. Varyani F, Argyriou K, Phillips F, Tsakiridou E, Moran G. Profile of tofacitinib in the treatment of ulcerative colitis: an evidence-based review of recent data. Drug Des Devel Ther. 2019;13:4091-105.

50. Moore H, Dubes L, Fusillo S, Baldassano R, Stein R. Tofacitinib therapy in children and young adults with pediatric-onset medically refractory inflammatory bowel disease. J Pediatr Gastroenterol Nutr. 2021;73(3):e57-e62.

51. López-Sanromán, Esplugues J, Domènech E. Pharmacology and safety of tofacitinib in ulcerative colitis. Gastroenterol Hepatol. 2021;44(1):3948.

52. Agrawal M, Kim E, Colombel J. JAK inhibitors safety in ulcerative colitis: practical implications. J Crohns Colitis. 2020;14(Suppl 2):S755-60.

53. Gisbert J, Chaparro M. Safety of new biologics (vedolizumab and ustekinumab) and small molecules (tofacitinib) during pregnancy: a review. Drugs. 2020;80(11):1085-100.

54. Crosby S, Schuh M, Caldera F, Farraye F. Vaccination of patients with inflammatory bowel disease during the COVID-19 pandemic. Gastroenterol Hepatol. 2021;17:18-30.

55. Fernández-Clotet $A$, Castro-Poceiro J, Panés J. Tofacitinib for the treatment of ulcerative colitis. Expert Rev Clin Immunol. 2018;14(11):881-92. 